\title{
Nuclear Reaction Taking Different Weight TARGET NUCLEUS
}

\author{
Rohit Gupta \\ Department of Physics, Institute of Basic Sciences, Dr. Bhim Rao Ambedkar University, \\ Khandari Campus, Agra (U.P.)-India.
}

\begin{abstract}
The nuclear reactions are very interesting and very big topic in research field, In this research paper we are describing about all types of nuclear reactions taking for light weight, medium weight and heavy weight target nucleus, which is only one types of target nucleus.

Here our interest how many types nuclear reactions occur or don't occur at taking for light weight, medium weight and heavy weight target nucleus. Finding the reason why it doesn't become to taking other weight target nucleus and why it becomes in those types of targets nucleus. So we can say it can become very interesting topic in field of finding new information about nucleus reactions to taking different types of targets nucleus.
\end{abstract}

\section{KEYWORDS}

Higgs boson, nuclear fission, nuclear fusion, incident particle, nuclear reaction and elementary particles.

\section{INTRODUCTION}

Here our interest taking only one nucleus which is in the form of light nucleus, medium nucleus and heavy nucleus in rest position and changed in the incident particles and its kinetic energy, we can mostly perform all types of nuclear reactions. But some nuclear reactions don't perform in light nucleus, medium nucleus and heavy nucleus, so we see here whose nuclear reactions don't perform in light nucleus, medium nucleus and heavy nucleus and why, getting its proper reason. Here we kwon that the light nucleus $(\mathrm{A}<40)$ mass number equal to or less than 40 , medium nucleus $(40<\mathrm{A}<150)$ mass number less than 150 and heavy nucleus $(\mathrm{A}>150)$ the mass number large than 200.

So here we take any light nucleus under 40, medium nucleus under 150 and heavy nucleus more than 150 .

\section{NUCLEAR REACTIONS}

In the nuclear reactions target nucleus and incident particle approach to each other, there are mainly some types of nuclear reactions also perform.

\subsection{ELASTIC REACTION}

When the incident particle don't enter field of targets nucleus it means don't changes inside nucleus and the target nucleus same as, this types of reactions isn't nuclear reactions, it's simply Elastic Reaction[2].

$$
{ }^{4} \mathrm{He}_{2}+{ }^{197} \mathrm{Au}_{79} \rightarrow{ }^{197} \mathrm{Au}_{79}+{ }^{4} \mathrm{He}_{2}
$$




\subsection{INELASTIC REACTION}

When the incident particles entre inside the field of target nucleus, incident particle loss energy on target nucleus and target nucleus reached in exited state, after loss exited energy the target nucleus come on ground state. It's called Inelastic Reaction [2].

$$
{ }^{7} \mathrm{Li}_{3}+{ }^{1} \mathrm{H}_{1} \rightarrow\left({ }^{7} \mathrm{Li}_{3}\right)^{*}+{ }^{1} \mathrm{H}_{1}
$$

\subsection{COMPOUND NUCLEAR REACTION}

When the incident particle break potential barrier of the target nucleus, the target nucleus and incident particle combined for very low time period also remains for a long time $\left(\cong 10^{-16} \mathrm{sec}\right)$ compared to small time $\left(\cong 10^{-22} \mathrm{sec}\right)$ and it is in highly exited state. Its called compound nuclear reactions.

There are many types of compound nuclear reactions basis of product nucleus and eject particle[1].

\subsubsection{Disintegration}

The incident particle strikes on the target nuclei, absorbed by it and a different particle is ejected by it, the product nucleus also different from target nucleus.

$$
{ }^{14} \mathrm{~N}_{7}+{ }^{4} \mathrm{He}_{2} \rightarrow\left({ }^{18} \mathrm{~F}_{9}\right) * \rightarrow{ }^{17} \mathrm{O}_{8}+{ }^{1} \mathrm{H}_{1}
$$

\subsubsection{Photo Disintegration}

The $\gamma$-ray particle also absorbed by the target nucleus, after absorbed $\gamma$ - ray the target nucleus reached in excited higher quantum state. If the energy is high enough, one or more particles may be reduce form it [1].

$$
{ }^{2} \mathrm{H}_{1}+\gamma \rightarrow\left({ }^{2} \mathrm{H}_{1}\right)^{*} \rightarrow{ }^{1} \mathrm{H}_{1}+{ }^{1} \mathrm{n}_{0}
$$

\subsubsection{Radioactive Capture}

When the incident particle break potential barrier of the target nucleus, the target nucleus and incident particle combined and product nucleus low exited state than only $\gamma$ photon is reduce by it.

$$
{ }^{26} \mathrm{Mg}_{12}+{ }^{1} \mathrm{H}_{1} \rightarrow\left({ }^{27} \mathrm{Al}_{13}\right)^{*} \rightarrow{ }^{27} \mathrm{~A}_{113}+\gamma
$$

\subsection{DiRECT REACTIONS}

Incident particle collides with target nucleus. In this process compound nucleus don't make and reaction performs very low time less than 10-22 sec. There are two types of Direct Reactions.

\subsubsection{Pickup Reaction}

In this type of reaction, the incident particle loses one or more nucleons on the target nucleus.

\subsubsection{Stripping Reaction}

$$
{ }^{63} \mathrm{Cu}_{29}+{ }^{2} \mathrm{H}_{1} \rightarrow{ }^{64} \mathrm{Cu}_{29}+{ }^{1} \mathrm{H}_{1}
$$

In this types of reaction, the target nucleus loss one or more particles at incident particle[5]. 


$$
{ }^{7} \mathrm{Li}_{3}+{ }^{1} \mathrm{H}_{1} \rightarrow{ }^{6} \mathrm{Li}_{3}+{ }^{2} \mathrm{H}_{1}
$$

\subsection{SPONTANEOUS DECAY}

The spontaneous reactions don't under control, in this Alfa and Beta particle decay form nucleus.

$$
{ }^{7} \mathrm{Li}_{3} \rightarrow{ }^{7} \mathrm{Be}_{4}+{ }^{0} \beta_{-1}+\bar{v}
$$

\subsection{SPALlation REACTIONS}

The incident particle capture by a heavy target nucleus, several particles of sufficient energy ejected from it, It is also known Spallation Reaction. The nuclear fission is a good example it.

$$
{ }^{235} \mathrm{U}_{92}+{ }^{1} \mathrm{n}_{0} \rightarrow{ }^{98} \mathrm{Zr}_{40}+{ }^{136} \mathrm{Te}_{52}+2{ }^{1} \mathrm{n}_{0}
$$

\subsection{High ENERGY REACTIONS}

In High Energy Reaction the energy range of incident particle about $150 \mathrm{MeV}$, the new kinds of particles (mesons, strange particles) are ejected from nucleons[1].

\subsection{HEAVY ION REACTIONS}

The nuclear reactions inducted by heavy ions $(A \geq 12)$, the reaction mechanism, such as coulomb excitation, elastic scattering, direct reactions and compound nucleus.

The nuclear reactions also process on the basis of incident practical, its energy and target nucleus such as[1].

\subsubsection{Transfer Reaction (Direct Reaction)}

$$
{ }^{14} \mathrm{~N}_{7}+{ }^{10} \mathrm{~B}_{5} \rightarrow{ }^{13} \mathrm{~N}_{7}+{ }^{11} \mathrm{~B}_{5}
$$

\subsubsection{Neutron Reactions}

$$
{ }^{93} \mathrm{Nb}_{41}+{ }^{12} \mathrm{C}_{6} \rightarrow{ }^{103} \mathrm{Ag}_{47}+{ }^{21} \mathrm{n}_{0}
$$

\subsubsection{Fission Spallation And Fragmentation Reaction}

$$
{ }^{27} \mathrm{~A}_{113}+{ }^{14} \mathrm{~N}_{7} \rightarrow\left({ }^{41} \mathrm{Ca}_{20}\right) * \rightarrow{ }^{1} \mathrm{H}_{1}+{ }^{21} \mathrm{n}_{0}+{ }^{38} \mathrm{~K}_{19}
$$

\section{NuClear REACtions ON THE BASIS OF WeIGHT OF TARget NuCleuS}

There we are using light weight target nucleus, medium weight target nucleus and heavy weight target nucleus.

\subsection{Light Weight Target Nucleus}

Let here we can take $7 \mathrm{Li} 3$ for target nucleus in nuclear reactions and nuclear reactions are perform as following.

\subsubsection{Elastic Reaction}

In light weight target nucleus, elastic reaction is possible.

$$
{ }^{7} \mathrm{Li}_{3}+{ }^{4} \mathrm{He}_{2} \rightarrow{ }^{7} \mathrm{Li}_{3}+{ }^{4} \mathrm{He}_{2}
$$




\subsubsection{Inelastic Reaction}

In light weight target nucleus, inelastic reaction is possible.

$$
{ }^{7} \mathrm{Li}_{3}+{ }^{4} \mathrm{He}_{2} \rightarrow\left({ }^{7} \mathrm{Li}_{3}\right)^{*}+{ }^{4} \mathrm{He}_{2}
$$

\subsubsection{Compound Nuclear Reaction}

In light weight target nucleus, all types compound nuclear reaction are possible.

\subsubsection{Disintegration}

$$
{ }^{7} \mathrm{Li}_{3}+{ }^{4} \mathrm{He}_{2} \rightarrow\left({ }^{11} \mathrm{Be}_{5}\right)^{*} \rightarrow{ }^{10} \mathrm{~B}_{5}+{ }^{1} \mathrm{n}
$$

\subsubsection{Photo Disintegration}

$$
{ }^{7} \mathrm{Li}_{3}+\gamma \rightarrow\left({ }^{7} \mathrm{Li}_{3}\right) * \rightarrow{ }^{4} \mathrm{He}_{2}+{ }^{3} \mathrm{H}_{1}
$$

\subsubsection{Radioactive Capture}

$$
{ }^{7} \mathrm{Li}_{3}+{ }^{4} \mathrm{He}_{2} \rightarrow\left({ }^{11} \mathrm{Be}_{5}\right)^{*} \rightarrow{ }^{11} \mathrm{Be}_{5}+\gamma
$$

\subsubsection{Direct Reactions}

In light weight target nucleus, both types direct reaction are possible.

\subsubsection{Pickup Reaction}

$$
{ }^{7} \mathrm{Li}_{3}+{ }^{2} \mathrm{H}_{1} \rightarrow{ }^{8} \mathrm{Li}_{3}+{ }^{1} \mathrm{H}_{1}
$$

\subsubsection{Stripping Reaction}

$$
{ }^{7} \mathrm{Li}_{3}+{ }^{1} \mathrm{H}_{1} \rightarrow{ }^{6} \mathrm{Li}_{3}+{ }^{2} \mathrm{H}_{1}
$$

\subsubsection{Spontaneous Decay}

In light weight target nucleus, spontaneous decay is possible.

$$
{ }^{11} \mathrm{Li}_{3} \rightarrow{ }^{11} \mathrm{Be}_{4}+{ }^{0} \beta_{-1}+\bar{v}
$$

\subsubsection{Spallation Reaction}

The spallation reaction is not possible in light weight target nucleus, it become only heavy weight target nucleus.

\subsubsection{High Energy Reactions}

It is possible taking light weight target nucleus. In it, the particles (meson, strange particles) produce by nucleons (proton, neutron).

\subsubsection{Heavy Ion Reactions}

It is not possible taking light weight target nucleus. 
International Journal of Recent Advances in Physics (IJRAP) Vol.6, No.1, February 2017

\subsubsection{Transfer Reaction (Direct Reaction)}

$$
{ }^{7} \mathrm{Li}_{3}+{ }^{6} \mathrm{Li}_{3} \rightarrow{ }^{9} \mathrm{Be}_{4}+{ }^{4} \mathrm{He}_{2}
$$

\subsubsection{Neutron Reaction}

$$
{ }^{7} \mathrm{Li}_{3}+{ }^{7} \mathrm{Li}_{3} \rightarrow{ }^{12} \mathrm{C}_{6}+{ }^{21} \mathrm{n}_{0}
$$

\subsubsection{Fission Spallation And Fragmentation Reaction}

$$
{ }^{27} \mathrm{Al}_{13}+{ }^{14} \mathrm{~N}_{7} \rightarrow\left({ }^{41} \mathrm{Ca}_{20}\right) * \rightarrow{ }^{38} \mathrm{~K}_{19}+{ }^{1} \mathrm{H}_{1}+{ }^{21} \mathrm{n}_{0}
$$

\subsection{Medium Weight Target Nucleus}

Let here we can take $\mathrm{Cu}$ for target nucleus in nuclear reactions and nuclear reactions are perform as following.

\subsubsection{Elastic Reaction}

Elastic reaction is possible taking medium weight target nucleus.

$$
{ }^{65} \mathrm{Cu}_{29}+{ }^{4} \mathrm{He}_{2} \rightarrow{ }^{65} \mathrm{Cu}_{29}+{ }^{4} \mathrm{He}_{2}
$$

\subsubsection{Inelastic Reaction}

Inelastic reaction is possible talking medium weight target nucleus.

$$
{ }^{65} \mathrm{Cu}_{29}+{ }^{4} \mathrm{He}_{2} \rightarrow\left({ }^{65} \mathrm{Cu}_{29}\right) *+{ }^{4} \mathrm{He}_{2}
$$

\subsubsection{Compound Nuclear Reaction}

In medium weight target nucleus, all types compound nuclear reaction are possible.

\subsubsection{Disintegration}

$$
{ }^{65} \mathrm{Cu}_{29}+1 \mathrm{H} 1 \rightarrow\left({ }^{66} \mathrm{Zn}_{30}\right) * \rightarrow{ }^{65} \mathrm{Zn}_{30}+{ }^{1} \mathrm{n}_{0}
$$

\subsubsection{Photo Disintegration}

$$
{ }^{65} \mathrm{Cu}_{29}+\gamma \rightarrow\left({ }^{65} \mathrm{Cu}_{29}\right)^{*} \rightarrow{ }^{65} \mathrm{Cu}_{29}+\gamma
$$

\subsubsection{Radioactive Capture}

$$
{ }^{65} \mathrm{Cu}_{29}+{ }^{0} \mathrm{n}_{1} \rightarrow\left({ }^{66} \mathrm{Cu}_{29}\right) * \rightarrow{ }^{66} \mathrm{Cu}_{29}+\gamma
$$

\subsubsection{Direct Reactions}

In medium weight target nucleus, both type direct reactions are possible.

\subsubsection{Pickup Reaction}

$$
{ }^{63} \mathrm{Cu}_{29}+{ }^{2} \mathrm{H}_{1} \rightarrow{ }^{64} \mathrm{Cu}_{29}+{ }^{1} \mathrm{H}_{1}
$$




\subsubsection{Stripping Reaction}

$$
{ }^{64} \mathrm{Cu}_{29}+{ }^{1} \mathrm{H}_{1} \rightarrow{ }^{63} \mathrm{Cu}_{29}+{ }^{2} \mathrm{H}_{1}
$$

\subsubsection{Spontaneous Decay}

In medium weight target nucleus, spontaneous decay is also possible.

$$
{ }^{64} \mathrm{Cu}_{29} \rightarrow{ }^{64} \mathrm{Zn}_{30}+{ }^{0} \beta_{-1}+\bar{v}
$$

\subsubsection{Spallation Reaction}

The spallation reaction is not possible in medium weight target nucleus, it become only heavy weight target nucleus.

\subsubsection{High Energy Reactions}

It is possible taking medium weight target nucleus. In it, the particles (meson, strange particles) produce by nucleons (proton, neutron).

\subsubsection{Heavy Ion Reactions}

In medium weight target nucleus, several heavy ion reaction possible and several not possible. As transfer reaction (direct reaction) and neutron reaction is also possible but fission spallation and fragmentation reaction is not possible[8].

\subsubsection{Transfer Reaction (Direct Reaction)}

$$
{ }^{65} \mathrm{Cu}_{29}+{ }^{13} \mathrm{~N}_{7} \rightarrow{ }^{63} \mathrm{Cu}_{29}+{ }^{15} \mathrm{~N}_{7} \rightarrow\left({ }^{65} \mathrm{Zn}_{30}+{ }^{13} \mathrm{C}_{6}\right)
$$

\subsubsection{Neutron Reactions}

$$
{ }^{65} \mathrm{Cu}_{29}+{ }^{14} \mathrm{~N}_{7} \rightarrow{ }^{78} \mathrm{Kr}_{36}+{ }^{1} \mathrm{n}_{0}
$$

\subsubsection{Fission Spallation And Fragmentation Reaction}

It is no possible in medium weight target nucleus.

\subsection{Heavy Weight TARget NuCleuS}

Let here we can take Au for target nucleus in nuclear reactions and nuclear reactions are perform as following[8].

\subsubsection{Elastic Reaction}

Taking heavy weight target nucleus, elastic reaction is possible.

$$
{ }^{197} \mathrm{Au}_{79}+{ }^{4} \mathrm{He}_{2} \rightarrow{ }^{197} \mathrm{Au}_{79}+{ }^{4} \mathrm{He}_{2}
$$


International Journal of Recent Advances in Physics (IJRAP) Vol.6, No.1, February 2017

\subsubsection{Inelastic Reaction}

Taking heavy weight target nucleus, inelastic reaction is possible.

\subsubsection{Compound Nuclear Reaction}

Taking heavy weight target nucleus, all types compound nuclear reaction is possible.

\subsubsection{Disintegration}

$$
{ }^{197} \mathrm{Au}_{79}+{ }^{3} \mathrm{H}_{1} \rightarrow\left({ }^{200} \mathrm{Hg}_{80}\right) * \rightarrow{ }^{199} \mathrm{Hg}_{80}+{ }^{1} \mathrm{n}_{0}
$$

\subsubsection{Photo Disintegration}

$$
{ }^{197} \mathrm{Au}_{79}+\gamma \rightarrow\left({ }^{197} \mathrm{Au}_{79}\right)^{*} \rightarrow{ }^{197} \mathrm{Au}_{79}+\gamma
$$

\subsubsection{Radioactive Capture}

$$
{ }^{197} \mathrm{Au}_{79}+{ }^{1} \mathrm{n}_{0} \rightarrow\left({ }^{198} \mathrm{Au}_{79}\right)^{*} \rightarrow{ }^{198} \mathrm{Au}_{79}+\mathrm{y}
$$

\subsubsection{Direct Reactions}

Taking heavy weight target nucleus, all type direct reaction is possible.

\subsubsection{Pickup Reaction}

$$
{ }^{197} \mathrm{Au}_{79}+{ }^{2} \mathrm{H}_{1} \rightarrow{ }^{198} \mathrm{Au}_{79}+{ }^{1} \mathrm{H}_{1}
$$

\subsubsection{Stripping Reaction}

$$
{ }^{197} \mathrm{Au}_{79}+{ }^{1} \mathrm{H}_{1} \rightarrow{ }^{196} \mathrm{Au}_{79}+{ }^{2} \mathrm{H}_{1}
$$

\subsubsection{Spontaneous Decay}

Taking heavy weight target nucleus, spontaneous reaction is possible.

$$
{ }^{198} \mathrm{Au}_{79} \rightarrow{ }^{198} \mathrm{Hg}_{80}+{ }^{0} \beta_{-1}+\bar{v}
$$

\subsubsection{Spallation Reaction}

It is possible in some heavy weight target nucleus, but not for all, it is not possible in Au target nucleus.

The suitable example is

$$
{ }^{235} \mathrm{U}_{92}+{ }^{1} \mathrm{n}_{0} \rightarrow\left({ }^{136} \mathrm{U}_{92}\right) * \rightarrow{ }^{92} \mathrm{Kr}_{36}+{ }^{141} \mathrm{Ba}_{56}+{ }^{31} \mathrm{n}_{0}+\gamma(\text { energy })
$$

\subsubsection{High Energy Reactions}

It is possible taking heavy weight target nucleus. In it the particles (meson, strange particle) produce by nucleons (proton and neutron). 


\subsubsection{Heavy Ion Reactions}

In heavy weight target nucleus, several nuclear reactions are possible and several rarely possible like, transfer reaction (direct reaction) and neutron reaction is also possible but fission spallation and fragmentation reaction is rarely possible[9].

\subsubsection{Transfer Reaction (Direct Reaction)}

$$
{ }^{197} \mathrm{Au}_{79}+{ }^{14} \mathrm{~N}_{7} \rightarrow{ }^{196} \mathrm{Pt}_{78}+{ }^{15} \mathrm{O}_{8}
$$

\subsubsection{Neutron Reactions}

$$
{ }^{197} \mathrm{Au}_{79}+{ }^{12} \mathrm{C}_{6} \rightarrow{ }^{205} \mathrm{At}_{85}+{ }^{41} \mathrm{n}_{0}
$$

\subsubsection{Fission Spallation And Fragmentation Reaction}

$$
{ }^{235} \mathrm{U}_{92}+{ }^{1} \mathrm{n}_{0} \rightarrow\left({ }^{236} \mathrm{U}_{92}\right) * \rightarrow{ }^{98} \mathrm{Zr}_{40}+{ }^{136} \mathrm{Te}_{52}+{ }^{21} \mathrm{n}_{0}
$$

\section{THE RESUlT}

In this, nuclear reaction (light weight, medium weight and heavy weight target) several types nuclear reactions become or don't become. The name of nuclear reactions which don't become each one as spallation reactions, High energy, Heavy ions reaction, Fission, etc. and the any other nuclear reactions become each one.

Any nuclear fusion possible in light weight nucleus $(A>20)$, Nuclear fission rarely possible in medium weight nucleus and large possibility in heavy weight nucleus.

\section{CONCLUSiON}

The several types of nuclear reaction which don't become or become taking weight target nucleus. We can understand that type nuclear reaction and classified it, in two ways as, target nucleus dependent nuclear reactions and nondependent nuclear reactions. It is very useful phenomena in nuclear transmutation. This type we can take any target nucleus and see by nuclear reaction or table nuclear transmutation must do.

\section{REFERENCES}

[1] Nuclear Physics book D.C. Tayal (Himalaya Publishing House) ISO 9001:2008 Certified.

[2] Burhan, W.E."Nuclear Physics", McGraw Hill Co. New York.

[3] Roy R.R. And B.P. Nigam,"Nuclear Physics", John Wiley, New York, 1967.

[4] Enge, Harald A. "Introduction to Nuclear Physics", Addition -Wesley,1966.

[5] butler, S.T., "Nuclear Stripping Reaction”, John Wiley, New York, 1957.

[6] Condon E.U. and Hugh Odishaw, "Hand-book of Physics", McGraw Hill, 1967.

[7] Prashant Kumar,IEC College of Engineering and Technology, India."Nano Nuclear Reaction", International Journal of Emerging Research and Technology.

[8] MasreshaFeleke, "Some Basics of Nuclear Reactions", ADDIS ABABA UNIVERSITY, ADDIS ABABA, ETHIOPIA (June 2011). 
International Journal of Recent Advances in Physics (IJRAP) Vol.6, No.1, February 2017

[9] Ankit Gupta and Rustum Sengupta, DEI Dayalbagh Agra- 282005. "Analytical Study of Department of NuclearFusion Reactors As Potential Source of Energy in the Future".

[10] Steven B. Krivit and Jan Marwan, “A New Look at Low-Energy Nuclear Reaction Research" (Sep2009), Journal of Environmental Monitoring.

[11] Hans Henrik Knudsen, Department of Physics and Astronomy University of Aarhus, " Low Energy Nuclear Reaction, Exploratory Work on11B” (August-2008), Sodium Petit in Profundis, UniversitasArhusiensis.

\section{AUTHOR}

Rohit Gupta is a M.Sc. form Agra, College Agra from Physics. Rohit Gupta is in Department of Physics, Institute of basic Sciences, Dr. Bhim Rao Ambedkar University, Khandari Campus, Agra (U.P.)-India

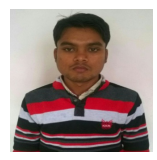

